\title{
Both atrioventricular nodal reentrant and atrioventricular reentrant tachycardias in one patient: a case report
}

\author{
Ivan Zeljković*, Šime Manola, Vjekoslav Radeljić \\ University Hospital Centre Sestre milosrdnice, Zagreb, Croatia
}

The two most common supraventricular tachycardias are typical (slow-fast) atrioventricular nodal reentrant tachycardia (AVNRT) and orthodromic atrioventricular reentrant tachycardia (AVRT) using accessory pathway (AP). Nowadays, a leading method in the treatment of supraventricular re-entrant tachycardias is radio-frequent (RF) catheter ablation during the electrophysiological (EP) study.

We report the case of a 46-year old woman on whom an EP study was performed due to the presence of paroxysmal supraventricular tachycardia documented in 12-lead ECG. During the EP study, at baseline, supraventricular tachycardia was induced very easily and it corresponded to orthodromic AVRT (frequency of 190-200/min) using concealed,

\section{Received: $30^{\text {th }}$ Apr 2014}

*Address for correspondence: Klinički bolnički centar Sestre milosrdnice, Vinogradska 29, HR-10000 Zagreb, Croatia.

Phone: 385-91-782-32-89

E-mail: ivanzeljkov@gmail.com left lateral AP (PPI - TCL = 100 ms; VAV pattern, preceding positive for AP). However, during the study, AVRT spontaneously converted to the typical slow-fast AVNRT (frequency $150 / \mathrm{min}$ ) using the slow AV node pathway (PPI - TCL >150 ms; VA < 30 ms; preceding negative for AP; jump noted at programmed pacing $(500+280 \mathrm{~ms})$ from right atrium as well as echo beat). During the EP study, a slow pathway was localized and then ablated using the RF catheter ablation. By performing the transseptal puncture of the interatrial septum, the left lateral wall AP was also successfully localized and then ablated using the RF catheter ablation. The patient remained asymptomatic for more than 6 months after the successful ablation procedures.

To the authors' best knowledge, the coexistence of AVRT and AVNRT is not reported in the literature.

KEYWORDS: accessory pathway, slow pathway, atrioventricular reentrant tachycardia, atrioventricular nodal reentrant tachycardia, catheter ablation.

CITATION: Cardiol Croat. 2014;9(5-6):216.

\section{Literature}

1. Padanilam BJ, Akula D, Vaughn P, Prystowsky EN. Successful elimination of concealed accessory pathway-mediated tachycardia by ablation of AV nodal slow pathway. $J$ Cardiovasc Electrophysiol. 2006;17(6):674-7.

2. Iyer R, Hameed S, Vora AM, Lokhandwala Y. Radiofrequency ablation: a cure for tachyarrhythmias. J Indian Med Assoc. 2000;98:684-7.

3. Orejarena LA, Vidaillet H Jr, DeStefano F, Nordstrom DL, Vierkant RA, Smith PN, et al. Paroxysmal supraventricular tachycardia in the general population. J Am Coll Cardiol. 1998;31:150-7. 